\title{
Using Fault Injection to Analyze the Scope of Error Propagation in Linux
}

\author{
Takeshi Yoshimura $^{1, a)}$ Hiroshi Yamada $^{2,3}$ Kenji Kono $^{1,3}$ \\ Received: October 19, 2012, Accepted: January 29, 2013, Released: April 24, 2013
}

\begin{abstract}
Operating systems (OSes) are crucial for achieving high availability of computer systems. Even if applications running on an operating system are highly available, a bug inside the kernel may result in a failure of the entire software stack. The objective of this study is to gain some insight into the development of the Linux kernel that is more resilient against software faults. In particular, this paper investigates the scope of error propagation. The propagation scope is process-local if the erroneous value is not propagated outside the process context that activated it. The scope is kernel-global if the erroneous value is propagated outside the process context that activated it. The investigation of the scope of error propagation gives us some insight into 1) defensive coding style, 2) reboot-less rejuvenation, and 3) general recovery mechanisms of the Linux kernel. For example, if most errors are process-local, we can rejuvenate the kernel without reboots because the kernel can be recovered simply by killing faulty processes. To investigate the scope of error propagation, we conduct an experimental campaign of fault injection on Linux 2.6.18, using a kernel-level fault injector widely used in the OS community. Our findings are (1) our target kernel (Linux 2.6.18) is coded defensively. This defensive coding style contributes to lower rates of error manifestation and kernel-global errors, (2) the scope of error propagation is mostly process-local in Linux, and (3) global propagation occurs with low probability. Even if an error corrupts a global data structure, other processes merely access to them.
\end{abstract}

Keywords: system dependability, software faults, error propagation, fault injection, rejuvenation

\section{Introduction}

Operating systems (OSes) are crucial for achieving high availability of computer systems. Kernel-level failures are known to occur less frequently compared with application-level failures, but they have a considerable impact on the overall availability of software systems. Even if applications running on an OS are highly available, bugs inside the kernel may result in a failure of the entire software stack; no application can continue to run on the crashed kernel.

Modern OSes are far from bug-free. Rich functionality of the OSes makes it harder to eliminate all the bugs before shipping. Although the advances in debugging tools, software testing methodologies, static analysis, and formal methods are tremendous, there are many software faults in production-quality OSes. According to Palix et al. [1], the rate of introduction of bugs continues to rise even in Linux 2.6.

The objective of this study is to gain some insight into design of the Linux kernel that is resilient against software faults. To this end, it is critically important to understand Linux kernel behaviors under software faults. We introduce the concept of the scope of error propagation. The propagation scope is process-local if the erroneous value is not propagated outside the process context that activated it. The scope is kernel-global if the erroneous value

\footnotetext{
Keio University, Yokohama, Kanagawa 223-8522, Japan

Tokyo University of Agriculture and Technology, Fuchu, Tokyo 1830057, Japan

JST CREST, Chiyoda, Tokyo 102-0076, Japan

a) yos@sslab.ics.keio.ac.jp
}

is propagated outside the process context that activated it. To the best of our knowledge, no existing work investigated the scope of error propagation.

This distinction between process-local and kernel-global errors is significant. If most errors are process-local, the kernel can recover from most errors simply by killing and revoking the resources of the faulty process. This implies that the Linux kernel can be partially rejuvenated without rebooting the entire OS because the kernel does not need to verify every kernel state. Note that this error recovery still requires further investigation. For example, killing a faulty process as error recovery can cause deadlocks. However, hundreds of experiments in our prior work show that the Linux can run reliably after killing a faulty process when the error is process-local [2]. If most errors are kernelglobal, the recovery becomes hopeless because corrupted global data structures must be recovered to continue processing. In this case, a mechanism isolating propagated errors should be developed rather than recovery mechanisms.

To investigate the scope of error propagation, a series of fault injection experiments is conducted. The fault injector used in our experiments is the existing one [3] that is widely used to evaluate the OS dependability in the OS research community [4], [5], [6], [7], [8]. It focuses on the emulation of low- and high-level software faults, including ones specific to OS kernels.

The distinguished feature of our study is threefold. First, we investigate the "scope" of error propagation; an error caused by a software fault propagates outside or confines within the context of the faulty process. Second, we focus on low- and 
high-level software faults, while the primary target of previous work [9], [10], [11] is low-level hardware faults such as flipping bits in memory. Flipping bits in memory can indirectly emulate low-level programming faults, however, high-level programming faults such as argument faults are neither emulated directly nor considered enough. Third, we show the detailed analysis of faults that are activated but do not manifest themselves.

This work investigates only corrupted data structures as errors although there are various kinds of other errors. For example, real bugs inside the Linux kernel can cause deadlocks and memory leaks. This work focuses only on whether the erroneous value propagation is confined in the faulty context of a process or not. Our contributions are 1) to introduce the concept of the error propagation scope, 2) to show an experimental result of the propagation scope by using fault injection technique, and 3) to show some insight obtained through the fault injection experiment.

In our fault injection experiments, 864 faults are injected into Linux 2.6.18 and 20\% of the injected faults are activated. Error propagation is investigated by using a built-in kernel debugger. The major findings include:

- Our target kernel (Linux 2.6.18) is coded in a defensive way. It frequently checks the integrity of function arguments, return values, and other important variables. This style of defensive coding contributes to lower rates of error manifestation (36\% of the fault activation) and kernel-global errors (16\% of the failures).

- The scope of error propagation is mostly process-local in Linux ( $84 \%$ of the failures). This implies that the Linux can be rejuvenated without reboots with high probability. Since an error is not propagated to other process contexts, the kernel can be recovered to a consistent state simply by revoking the context of a faulty process.

- Global propagation of errors occurs with low probability. Interestingly, even if a global data structure is corrupted, the corrupted data cannot be accessed from the other processes in our experiment. This is because the faulty process crashes with a lock acquired inside a critical section.

We use some terms throughout this paper, derived from definition by Patterson [12] and other dependable computing researches. The readers familiar with the terms can skip this paragraph. We distinguish between a fault, an error and a failure. A fault is a defect in a component. This work focuses on defects which derive from programming mistakes (i.e., bugs) in the $\mathrm{C}$ language code of the Linux kernel. "Inject a fault" means introducing a defect intentionally in a component. "Activate a fault" means that a fault becomes effective; a faulty region in a component is executed. An error is a state which is changed by a defect. An error is caused by a fault activation. An error often propagates from one component to another, thereby creating new errors. "Manifest a fault or an error" means that a defect affects a delivered service by triggering a failure, which is deviant behavior (e.g., a kernel crash).

The rest of this paper is organized as follows. Section 2 describes related work. Section 3 explains the software fault injector we used. Section 4 describes our methodology of the experimental campaign of fault injection. Section 5 reports our experimental results. Section 6 discusses the directions towards more resilient structure of the Linux kernel. Section 7 concludes this paper.

\section{Related Work}

Understanding the kernel behavior under fault manifestation can be an aid for kernel developers to improve the kernel dependability or develop the mechanisms for kernel recoveries. OS kernel behavior under fault manifestation has been widely examined.

Software-implemented fault injection (SWIFI) has been conducted with emphasis placed on the different aspects of fault manifestation to better understand the kernel behavior under fault manifestation. This work focuses on the "scope" of error propagation (i.e., process-local or kernel-global), while previous work focuses on other aspects of error propagation than the scope of error propagation. This work is extended from our previous work [2], [13] and reports more detailed results.

Gu et al. [9] use SWIFI to characterize Linux behaviors under error manifestation. Their analysis shows that crash latencies are within 10 cycles in most cases and also shows how an error propagates between OS subsystems. Our concern in this paper is that an error propagates beyond the boundary of the process context. Pham et al. [14] use SWIFI for their framework that automates to validate the robustness of virtualized environments based on KVM or Xen hypervisors.

Chen et al. [11] and another paper from Gu et al. [10] investigate behavioral difference caused by different combinations of CPU architectures and OSes (five combinations of CPU and OSes are investigated in total). These studies indicate a good insight into the design principles of CPU architectures and OSes that are resilient to faults. However, these studies do not address the scope of error propagation. The fault models considered in these studies are device-level transient faults, while the fault model used in our study is low- and high-level programming errors.

The techniques used in SWIFI are evolving. G-SWFIT precisely emulates general software faults by mutating binary executable code [15]. According to the analysis by Cotroneo et al. [16], G-SWFIT improves the fault injection accuracy. Unfortunately, G-SWFIT does not emulate faults that are specific to Linux kernels. So, we use another fault injector that is widely used in the OS community.

Aside from fault injection studies, software bugs in the production-quality OSes such as Linux are extensively examined. Chou et al. [17] apply a static analyzer to Linux versions 1.0 through Linux 2.4.1 to study the trend of software bugs in the Linux kernels. Palix et al. [1] are the most recent follow-up that investigates Linux versions 2.6.0 to 2.6.33. The primary goal of these studies is to identify the distribution and lifetime of certain kinds of faults in the Linux kernels.

To mitigate the impact of kernel failures, numerous mechanisms for kernel recovery have been proposed. Swift et al. [4], [5] propose a kernel mechanism of managing and recovering from device driver failures. Otherworld [6] restarts the kernel without discarding applications memory states when the kernel crashes. Phase-based Reboot [7] shortens downtime involved in rebootbased recovery. 


\section{Fault Injector}

We investigate the scope of error propagation in a commodity OS kernel (i.e., Linux) under software fault manifestation. To this end, an experimental campaign of fault injection is conducted in Linux to examine how it reacts to the injected faults. The injector that is originally obtained from the Nooks web site is ported to the $x 86$ Linux 2.6.18 kernel. This section briefly describes the injector and the fault types it injects.

\subsection{Overview}

The injector [3] emulates low- and high-level programming bugs specific to OS kernels. It changes individual instructions in the kernel text segment. These faults are intended to approximate the assembly-level manifestation of real C-level programming errors. For example, the injector emulates missing initialization by deleting instructions that are responsible for variable initialization. The details of the faults are described in Section 3.2.

The injector is widely used to evaluate and validate recovery mechanisms in the OS research community. For example, it was used to evaluate the fault tolerance of the file system cache [3], recovery mechanisms for device drivers [4], [5], a kernel mechanism for applications to survive OS crashes [6], and a quick mechanism for reboot-based recovery [7].

The injector runs in the kernel and provides a system call interface to specify the parameters of fault injection. It rewrites the binary code of the running kernel to inject each type of fault. The injector disassembles the binary of a randomly selected function in the kernel text segment. Since the faults injected by our injector are context-dependent, it analyzes the disassembled code and searches for proper locations to which each type of fault can be injected.

\subsection{Injected Faults}

The injector emulates 10 types of faults. These faults range from low-level hardware faults to high-level software faults. Since our primary concern is in programming errors, we omitted 3 types of faults that emulate hardware faults. So, 7 types of faults are injected in our experiments. For ease of understanding, Table 1 lists some examples of injected faults at the C-language level, although the injection is done at the binary level.

- INIT FAULT: INIT fault creates a situation where the initialization of variables is missed. To create such a situation, the injector deletes instructions responsible for initializing a variable by copying a constant value. More concretely, it deletes an instruction that assigns an immediate value to the

Table 1 C-Language Level View of the Injected Software Faults. This table shows examples of the injected faults at the C-language level.

\begin{tabular}{c|c|c}
\hline \hline Fault & Before & After \\
\hline INIT & int $\mathrm{x}=\boldsymbol{\theta} ;$ & int $\mathrm{x} ;$ \\
\hline DST\&SRC & $\mathrm{x}+=1 ;$ & $\mathrm{x}+=2 ;$ \\
\hline PTR & ptr $=$ list->prev; & ptr $=$ list->next; \\
\hline BRANCH & if $(\mathrm{x} !=0)$ return; & return; \\
\hline INVERSE & if $(\mathrm{x}==0)$ & if $(\mathrm{x} !=0)$ \\
\hline INTERFACE & func $(1,2,3) ;$ & func $(1,214,3) ;$ \\
\hline IRQ & local_irq_restore ()$;$ & deleted. \\
\hline
\end{tabular}

address lower than the stack pointer.

- DST\&SRC FAULT: This fault corrupts assignment statements. It creates a situation where the assignment is incorrect due to a programming error. To do this, the injector corrupts the value of the source or the destination by flipping the bits of the value.

- PTR FAULT: This fault emulates pointer corruption by corrupting the addressing bytes of instructions. The injector either flips a bit within the addressing-form specifier byte (ModR/M) or the scale, index or base (SIB) byte following the instruction opcode.

- BRANCH FAULT: This fault emulates an incorrect control flow by deleting a jump instruction involved in the conditional statement. By doing this, the injector emulates branch errors and error handling faults.

- INVERSE FAULT: The injector also reverses the predicates of conditional statements to inject incorrect control flows. For example, this fault changes " $\mathrm{je}$ " into "jne" to reverse the predicate.

- INTERFACE FAULT: This fault corrupts one of the arguments passed to a procedure. To create this situation, the injector deletes an instruction that copies a value at an address below the base pointer to registers or memory. For example, the injector can change the call foo $(a, b)$ to $f o o(X, b)$, where $\mathrm{X}$ is a corrupted value, by deleting the instruction that copies a to a register or memory.

- IRQ FAULT: When an IRQ fault is injected, the injector creates a situation where a kernel developer forgets to enable interrupts after disabling them. The injector removes local_irq_restore() calls. When a call to local_irq_restore() is removed, the interrupt mask is not restored and thus the disabled interrupts continue to be disabled.

\section{Methodology}

To investigate the scope of error propagation, we track the Linux kernel behavior when an injected fault is activated. The kernel version of Linux we use is 2.6.18.8. 864 faults are injected one-by-one in our experiments. The number of fault injection for each fault type is described in the figures of the next section. To track how the Linux kernel reacts to the injected faults, we take the following steps manually:

(1) Injecting a fault: We request the injector to inject a fault. In our experiments, only the text segment is modified to inject faults as our target is programming errors. The injected erroneous instructions may corrupt data in heap or stack. To trace the kernel execution after the fault is activated, we set a breakpoint at the instruction to which a fault is injected. When the breakpoint is hit, the control is transferred to KDB, a built-in kernel debugger for Linux. We do not inject faults into the KDB code.

(2) Running a workload: The workload that we use to activate injected faults is to restart all the daemons. Since the daemons extensively issue system calls, the kernel code runs very frequently while the daemons are restarted.

(3) Tracing error propagation: After the fault is activated, the CPU is set into the single-step execution mode to take a trace of 
every instruction. Using the execution trace, the scope of error propagation is analyzed in the same way as taint analysis. If the injected fault produces an erroneous value, the value is marked as an "error." When the value marked as an "error" is used to calculate another value, the calculated value is also marked as an "error." If the value marked as an "error" is used in the prediction of conditional branches, all the values updated in the taken clause are marked as an "error." If no value marked as an "error" is written to a heap, the error is concluded to be process-local. Otherwise, the error is concluded to be kernel-global. The kernel execution is tracked until kernel failures (e.g., kernel panic). If all the daemons are restarted successfully, the error is classified into "not manifested."

The previous study using fault-injection shows crash latencies are within 10 cycles in most cases [9]. However, this ignores, for instance, aging-related failures. The workload used in this experiment runs only within less than one minute. To cover these failures, we also show the brief discussions of not-manifested errors in Section 5.4.

Note that error propagation is investigated at the assembly code level in our experiments, although this section describes the analysis of error propagation at the source code level for readability. Error propagation can be analyzed more precisely if it is analyzed at the assembly level. For example, compilers generate optimized code that shares common expressions. Supposed that there are two expressions: $\mathrm{x}=\mathrm{a}+\mathrm{b}$ and $\mathrm{y}=(\mathrm{a}+\mathrm{b}) * \mathrm{c}$. If a fault is injected into the former $\mathrm{a}+\mathrm{b}$, it propagates to the latter.

\section{Experiments}

\subsection{Experimental Setup}

Our experimental campaign of fault injection is carried out on VMWare Workstation 7.1.2 running on Windows 7. We run Fedora 8 (Linux 2.6.18.8) in a guest virtual machine that consists of 1 CPU, 1 GB of memory and $20 \mathrm{~GB}$ hard disk drive. The host $\mathrm{CPU}$ is $2.53 \mathrm{~GB}$ Core 2 Extreme CPU. The kernel configuration is default. Note that the failures encountered in these experiments are triggered by injected faults, not bugs in the Linux kernel, although real bugs inside the kernel can trigger failures during our experiments.

\subsection{Overall Results}

Figure 1 shows the overall results of our fault injection experiments. In total, 864 faults are injected and $20 \%$ of them are activated. Figure 1 (c) shows the ratio of fault injection sites in terms of the directory name in the kernel source code. Figure 1 (d) shows the ratio of fault activation sites in terms of the directory name. Our fault injector selects injected location randomly from the kernel text segment, so the ratio depends on the size of each subsystem that is built in the kernel. INIT, INTERFACE and IRQ faults cover relatively small number of directories because their target instructions (initializing with the base register and restoring the flags) are limited compared to other faults. These figures show our fault injection campaign covers all the common kernel subsystems. This result implies that injecting more errors will show the same categorization trend of errors.

Figure 1 (b) shows the failures which are observed after the fault activations. Segmentation failures ("SEG_F” in Fig. 1 (b)) are caused in $20 \%$ of the fault activations. They occur when the kernel attempts to access illegal pages. Intentional kernel crashes caused by BUG_ON are observed in 6\% ("BUG_ON" in Fig. 1 (b)). BUG_ON denotes a situation where Linux BUG_ON macro, similar to $\mathrm{C}$ assert, detects an erroneous state in the kernel. The other failures are panic, hangs and fail silence violations ("FSV" in Fig. 1 (b)). $64 \%$ of the activated faults do not manifest themselves.

Figure 2 (a) summarizes the result of the scope analysis. $84 \%$ of the manifested errors are process-local, while $16 \%$ of them are kernel-global. BRANCH and IRQ faults are not propagated outside a faulty context, while INTERFACE faults are the highest rate of kernel-global errors. IRQ faults and their error propagation are described in Section 5.3.1 (b). INTERFACE faults tend to corrupt the linked lists for kernel descriptors. The typical case is shown in Section 5.3.2. The manifested BRANCH faults tend to be injected to a branch instruction for a NULL check. Checked pointers are usually used in the clauses. So, a crash tends to occur soon after the fault activation. The short crash latency leads to the errors which are confined within a faulty context.

Figure 2(b) summarizes observed failures in terms of their error propagation scope. These segmentation failures occur in both propagation scopes with the highest probability out of all the observed failures (56\% of all the manifested errors). All of the fail silence violations and BUG_ON are caused only by process-local errors. This result implies that BUG_ON effectively prevents global propagation in the kernel as described in Section 5.3.1.

This experimental environment uses ext3 file system, which is the default configuration of Fedora 8. Real bugs inside the kernel might destroy the file system structure. However, any experimental results do not show such cases.

\subsection{Scope Analysis}

This section shows the detailed analysis of kernel traces. Error propagation are thoroughly examined in terms of their scope.

\subsubsection{Process-local Errors}

Table 2 shows typical examples of each failure type caused by process-local errors. The table lists an injected fault type, a memory address where the fault is injected, the location at the source code level, and the instructions and C-code before/after the fault injection.

(a) Segmentation Failure: As shown in Fig. 2 (b), 56\% of the process-local errors lead to segmentation failures. Table 2 (a) shows the detail of a typical case that leads to a segmentation failure. In this case, a null pointer is passed to a function that expects the passed pointer not to be null. This fault is injected by INVERSE FAULT. More concretely, the code

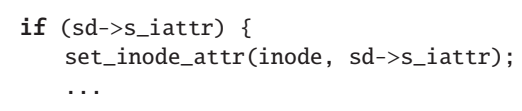

In the original code, set_inode_attr is called only when 


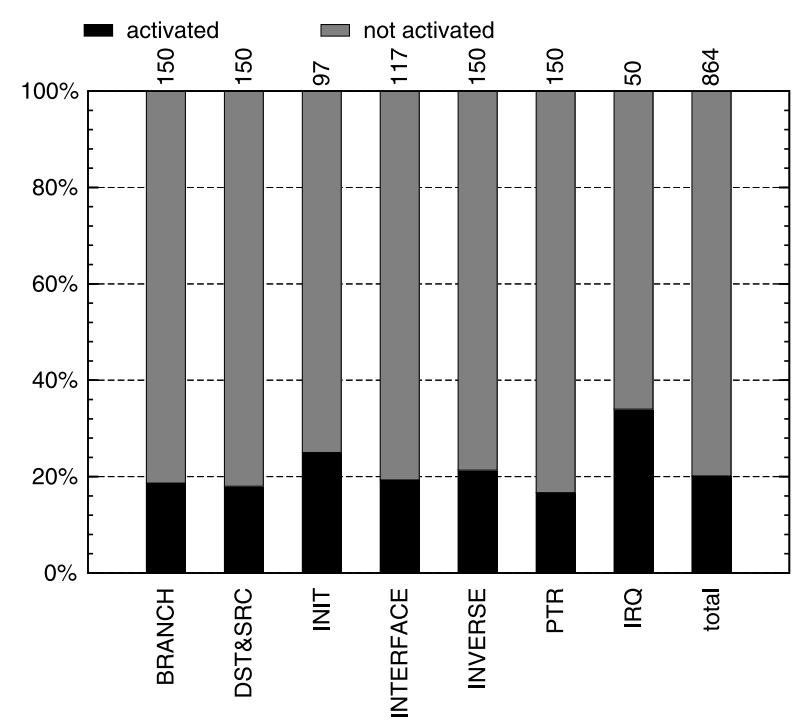

(a) Activated/Not Activated Faults

This figure shows the relative frequency with which injected faults are activated or not. The number at the end of each bar represents the total number of injected faults.

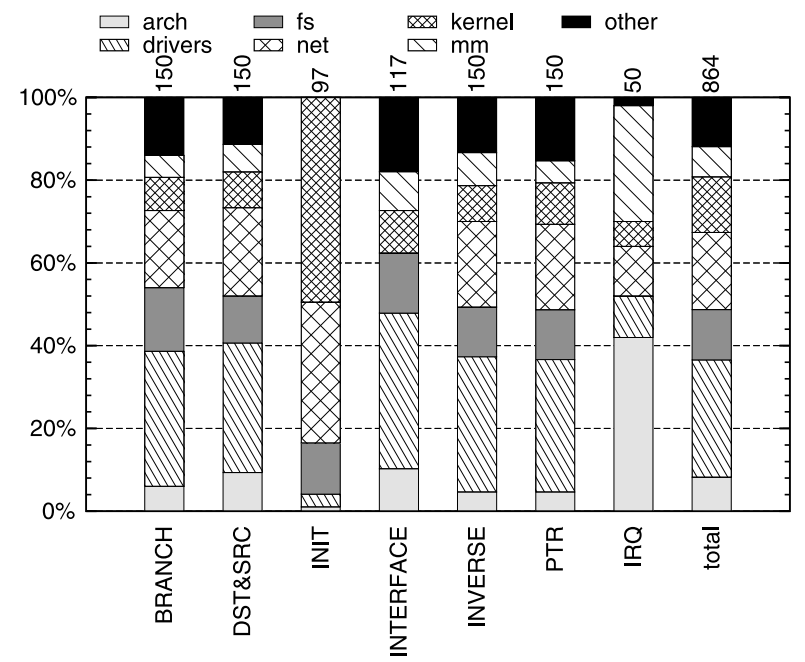

(c) Fault Injection Sites

This figure shows the relative frequency on which directory in the kernel source code faults are injected. The number at the end of each bar represents the total number of injected faults.

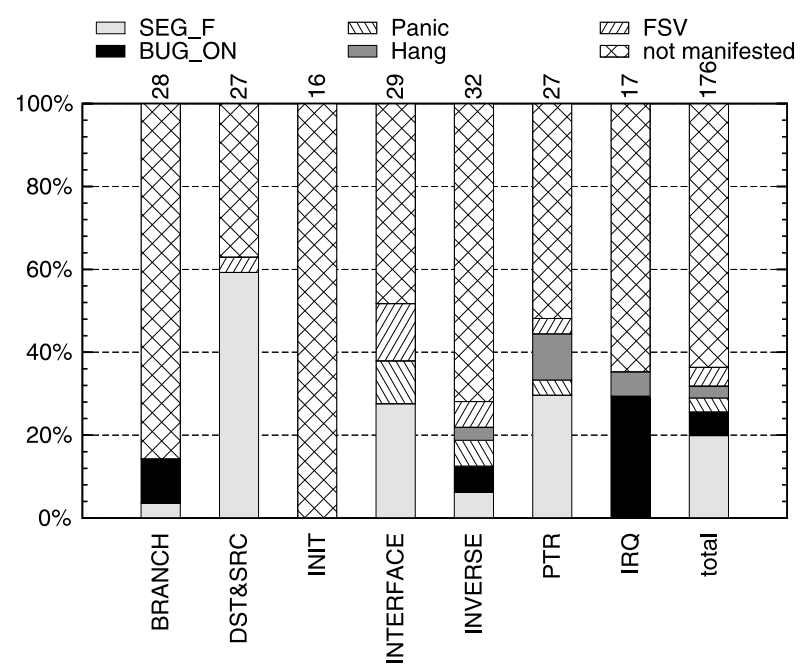

(b) Observed Failures

This figure shows the relative frequency of not-manifested errors and the failure categories of manifested errors. The number at the end of each bar represents the total number of activated faults.

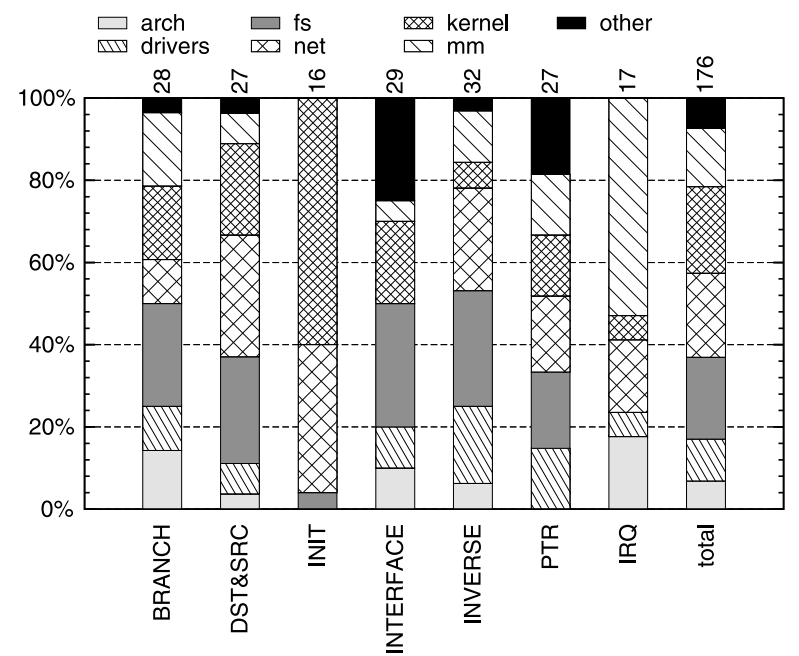

(d) Fault Activation Sites

This figure shows the relative frequency on which directory in the kernel source code faults are activated. The number at the end of each bar represents the total number of activated faults.

Fig. 1 Overall Fault Injection Results.

sd->s_iattr is not NULL. However, set_inode_attr is called when sd->s_iattr is NULL in the modified code. As a result, parameter iattr in set_inode_attr becomes NULL as shown below. The dereference of iattr causes a segmentation fault.

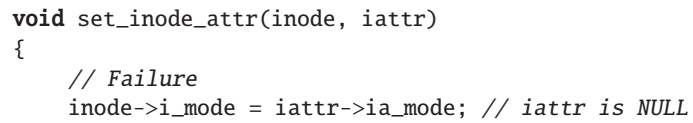

In this case, a null pointer is passed across function calls but no global data structures are updated with the incorrect null pointer. Thus, the scope of error propagation is process-local.

(b) BUG_ON: As shown in Fig. 2(b), 19\% of the processlocal errors lead to BUG_ON. An example of this failure is caused by IRQ FAULT, which removes the call to local_irq_restore to forget to enable disabled interrupts. After this fault is acti- vated, the kernel continues to run with the interrupts disabled. Meanwhile, lookup_bh_lru(bdev, block, size) is invoked. This function eventually calls check_irqs_on, which executes BUG_ON (irq_disabled()). Since the interrupts are disable here (if the fault is not injected, the interrupts are enable here), BUG_ON macro successfully detects this incorrect status of interrupts.

This experimental result suggests that BUG_ON macro is effective to prevent global error propagation. If BUG_ON is not used to check the status of interrupts, blocking functions are called with the interrupts disabled and thus, the deadlock or other serious situations would be caused. In the current versions of Linux, BUG_ON macro is inserted manually according to the developers' experiences and intuitions. We expect that more systematic methods are required in order to help the developers insert BUG_ON macros correctly. 


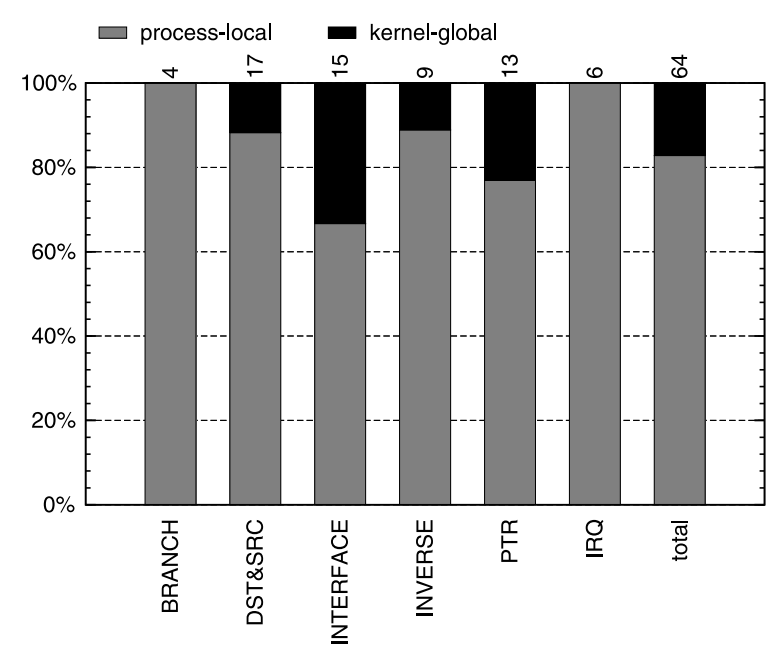

(a) Error Propagation Scope

This figure shows the relative frequency of process-local or kernelglobal errors. The number at the end of each bar represents the total number of investigated errors.

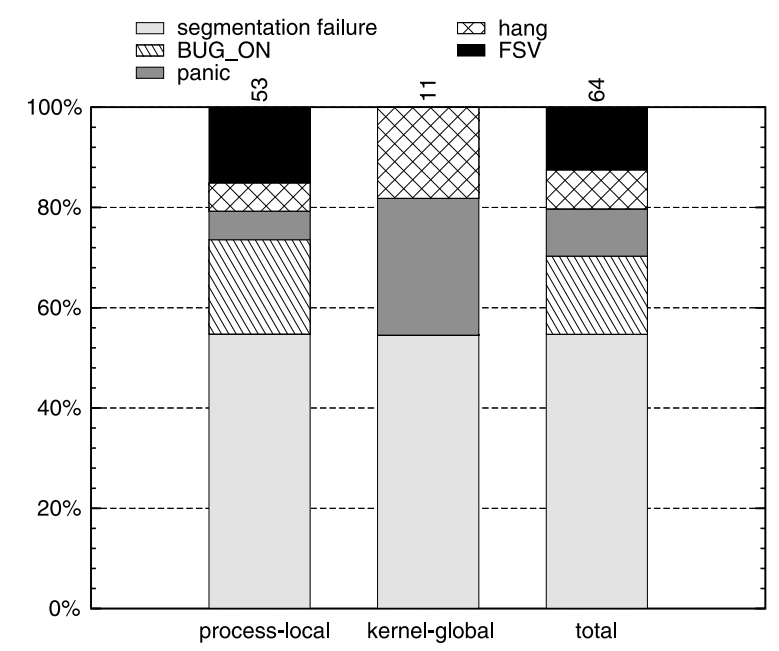

(b) Failure Type by Scope

This figure shows the relative frequency with which the kernel causes different failure categories after fault activations. The number at the end of each bar represents the total number of investigated errors.

Fig. 2 Overall Result of Scope Analysis.

(c) Panic: As shown in Fig. 2 (b), 6\% of the process-local errors cause kernel panic. Table 2 (c) shows a typical example of panic. In this case, a fault is injected into an interrupt handler. More concretely, an argument to function neigh_update is corrupted and thus the address of neigh->dev, which is calculated from the corrupted argument, becomes an incorrect value. As a result, the first access to neigh->dev causes a segmentation failure. Since this code is executed in an interrupt handler, the kernel invokes panic instead of causing a segmentation failure. Interrupt contexts temporarily use a kernel stack of the current process's kernel context in the Linux kernel. We do not observe any structural differences between interrupt and processes' contexts when we analyze the error propagation scope. Therefore, we regard the context as a process's context and the error confined in it is process-local.

(d) Fail silence violation: There are $15 \%$ of the process-local errors that lead to fail silence violation as shown in Fig. 2 (b). In our experiments, Fail silence violations often derive from kernel error detections. Despite their correctness, the kernel starts to handle the detected errors by the usual error processing manner. Besides, such error processing tends to simply abandon the current processing and return a corresponding erroneous value (e.g., EINVAL), therefore, global data structures are merely updated before fail silence violations occur. In our experiments, we do not observe any kernel-global errors that lead to fail silence violations.

The following is a typical example of fail silence violation. In this example, the injected fault generates a situation in which there is no unused network sockets. So, the Linux kernel considers no network sockets can be created. The following is simplified code for explanation. The original code

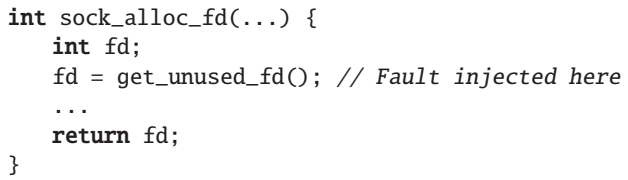

is modified to

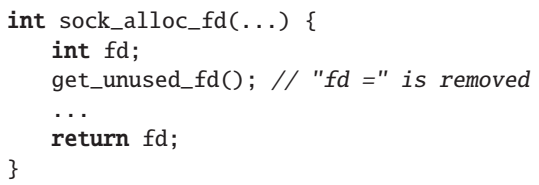

In the modified code, $\mathrm{fd}$ is not initialized. In our experiment, uninitialized $f d$ happens to be negative. As a result, sock_alloc_fd returns a negative value to its caller. The caller is:

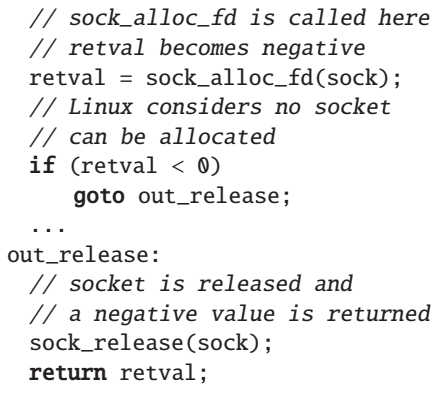

In the above code, the Linux kernel considers there is no room to create a new socket because sock_alloc_fd returns a negative value. As a result, a process cannot create a new socket even though there is enough room to create new sockets.

(e) Hang: As shown in Fig. 2(b), there are 5\% cases in which the Linux kernel hangs up. The typical example is shown in Table 2 (e). In this example, IRQ FAULT is injected into do_softirq which schedules pending software interrupts. When do_softirq returns, the kernel hangs immediately without dumping the stack trace. So, we cannot trace the kernel behavior using KDB. Since we can not determine from the source code which function is executed after do_softirq returns, further information cannot be obtained in this case.

\subsubsection{Kernel-global Errors}

$16 \%$ of the errors are kernel-global as shown in Fig. 2 (a), while all the other errors are process-local. Some of the process-local 
Table 2 Faults causing process-local errors.

(a) Segmentation Failure

\begin{tabular}{l|l}
\hline \hline Fault & INVERSE FAULT \\
\hline Memory Address & sysfs_new_inode+0x5c \\
\hline Code Location & fs/sysfs/inode.c, line:134 \\
\hline Original Instruction & je sysfs_new_inode+0x97 \\
\hline Modified Instruction & jne sysfs_new_inode+0x97 \\
\hline Original Code & if (sd->s_iattr) \{ \\
\hline Modified Code & if (!sd->s_iattr) \{ \\
\hline
\end{tabular}

(b) BUG_ON

\begin{tabular}{l|l}
\hline \hline Fault & IRQ FAULT \\
\hline Memory Address & kfree+0x5f \\
\hline Code Location & $\mathrm{mm} / \mathrm{slab.c} \mathrm{line:} \mathrm{3463}$ \\
\hline Original Instruction & push \%esi popf \\
\hline Modified nstruction & nop nop \\
\hline Original Code & local_irq_restore(flags); \\
\hline Modified Code & deleted \\
\hline
\end{tabular}

(c) Panic

\begin{tabular}{l|l}
\hline \hline Fault & INTERFACE FAULT \\
\hline Memory Address & neigh_update+0x1ed \\
\hline Code Location & $\begin{array}{l}\text { net/core/neighbour . c } \\
\text { line: 894-895 }\end{array}$ \\
\hline Original Instruction & mov Qxc(\%ebp), \%eax \\
\hline Modified Instruction & nop nop nop \\
\hline Original Code & $\begin{array}{l}\text { void (*update) }(. . .)= \\
\text { neigh->dev-> } \\
\text { header_cache_update; }\end{array}$ \\
\hline \multirow{2}{*}{ Modified Code } & $\begin{array}{l}\text { void (*update) }(. . .)= \\
\text { (struct netdevice *) }(\operatorname{\theta x} 6)-> \\
\text { header_cache_update; }\end{array}$ \\
\hline
\end{tabular}

(d) Fail silence violation

\begin{tabular}{l|l}
\hline \hline Fault & SRC\&DST FAULT \\
\hline Memory Address & sock_alloc_fd+0xb \\
\hline Code Location & net/socket.c, line:380 \\
\hline Original Instruction & mov \%eax, \%ebx \\
\hline Modified Instruction & mov \%esp, \%ebx \\
\hline Original Code & fd $=$ get_unused_fd(); \\
\hline Modified Code & get_unused_fd(); \\
\hline
\end{tabular}

(e) Hang

\begin{tabular}{l|l}
\hline \hline Fault & IRQ FAULT \\
\hline Memory Address & do_softirq+0x48 \\
\hline Code Location & kernel/softirq.c, line: 215 \\
\hline Original Instruction & push \%esi popf \\
\hline Modified Instruction & nop nop \\
\hline Original Code & local_irq_restore(flags); \\
\hline Modified Code & deleted \\
\hline
\end{tabular}

errors propagate across multiple function calls but the propagations are limited to function arguments, return values, and local variables. This is probably because global data structures, shared among multiple processes, are used to store stable, consistent states rather than transient, temporary states. Experienced programmers like Linux developers write defensive code that checks data integrity and/or confirms the assumptions on function arguments. A data is checked again and again before it is written to global data structures.

Table 3 shows the detail of a representative kernel-global error. In this case, a fault is injected into a function that manages redblack trees, a type of self-balancing binary search tree, used for storing sortable key-value pairs. More specifically, INTERFACE FAULT is injected into the call to __rb_erase_color. Function __rb_erase_color takes three arguments: node, parent, and root whose types are all struct rb_node*. By the INTER-
Table 3 A Kernel-global error.

\begin{tabular}{l|l}
\hline \hline Fault & INTERFACE FAULT \\
\hline Memory Address & rb_erase+0x1e9 \\
\hline Function & lib/rbtree.c, line $: 178$ \\
\hline Original Instruction & mov $0 \times 0$ (\%ebp),\%ebx \\
\hline Modified Instruction & nop nop nop \\
\hline Original Code & node $=$ root $->$ rb_node $;$ \\
\hline Modified Code & node $=$ parent $->$ rb_right $;$ \\
\hline
\end{tabular}

Table 4 Summary of Not-Manifested Errors. This table shows the number of errors for each reason that activated errors do not manifest themselves. We conclude that an error does not manifest itself when one of these situation is observed during the tracing of error propagation. The untraceable cases are discussed in detail.

\begin{tabular}{l|r}
\hline \hline Reason & \# of errors \\
\hline Corrected & 8 \\
Not affecting & 10 \\
Error processing omitted & 18 \\
Incorrect warning & 4 \\
Almost correct operation & 15 \\
Aging & 6 \\
Lucky & 40 \\
Untraceable & 11 \\
\hline Total & 112 \\
\hline
\end{tabular}

FACE FAULT, argument node that should be root->rb_node is modified to parent->rb_right. As you can imagine from the arguments, __rb_erase_color manipulates tree structures in the heap. The incorrect argument leads to the corruption of the global tree structures. When the kernel traverses a broken red-black tree, it crashes due to segmentation fault. Since global data structures are corrupted by injected faults, the scope of this error is kernelglobal.

There is one important thing to be noted. The fault shown in Table 3 corrupts global data structures. However, the erroneous values are never propagated to other processes than the faulty one. Other processes can continue to run reliably because the error can be isolated. This is because the faulty process can hold a lock (more precisely, semaphore) for exclusive access to global data structures. If a faulty context does not release the lock, other processes cannot access the broken data structures; the corrupted data is never propagated to other processes. This example shows a deadlock leads to fail-stopping behavior although deadlock should be avoided. We found that deadlock prevents contexts from reading erroneous values, which may cause some incorrect kernel behavior like file system corruption. However, further research effort is required to apply this property of synchronization primitives to error recovery in practice.

\subsection{Not-Manifested Errors}

To understand Linux behaviors under software faults, it is critically important to analyze the reason why activated faults do not manifest themselves. As pointed out in many literatures, activated faults do not always manifest themselves. In our campaign of fault injection, These "not-manifested" errors are observed in $64 \%$ of the fault activations. If an error is corrected during the execution, the analysis aids in proposing defensive coding styles effective for kernels.

In our experiments, we trace error propagation with the kernel debugger until the kernel detects an error or we are sure of the error not manifesting itself. Table 4 shows the summary of the 
Table 5 Examples of Not-Manifested Errors.

(a) Corrected

\begin{tabular}{l|l}
\hline \hline Fault & INIT FAULT \\
\hline Memory Address & sched_setscheduler+0x44 \\
\hline Code Location & kernel/shed.c, line:4087 \\
\hline Original Instruction & movl \$Qxfffffff, Dxffffffec(\%ebp) \\
\hline Modified Instruction & nop nop ... nop \\
\hline Original Code & int oldpolicy $=-1 ;$ \\
\hline Modified Code & int oldpolicy; \\
\hline
\end{tabular}

(c) Error processing omitted

\begin{tabular}{l|l}
\hline \hline Fault & BRANCH FAULT \\
\hline Memory Address & follow_page+Qxd8 \\
\hline Code Location & mm/memory.c, line: 935 \\
\hline Original Instruction & je follow_page+Qx1aa \\
\hline Modified Instruction & nop nop ... nop \\
\hline Original Code & if (! ptep) goto out; \\
\hline Modified Code & deleted \\
\hline
\end{tabular}

(e) Almost correction operation

\begin{tabular}{l|l}
\hline \hline Fault & INIT FAULT \\
\hline Memory Address & schedule+0xd2 \\
\hline Code Location & kernel/sched.c, line:3341 \\
\hline Original Instruction & movl $0 \times 3 b 9 a c a 99,0 x f f f f f f(\%$ ebp) \\
\hline Modified Instruction & nop nop ... nop \\
\hline Original Code & run_time = NS_MAX_SLEEP_AVG; \\
\hline Modified Code & deleted \\
\hline
\end{tabular}

(g) Lucky

\begin{tabular}{l|l}
\hline \hline Fault & PTR FAULT \\
\hline Memory Address & tty_register_driver+0x6 \\
\hline Code Location & drivers/char/tty_io.c, line:3733 \\
\hline Original Instruction & mov 0x6c(\%esi),\%eax \\
\hline Modified Instruction & mov 0x6d(\%esi),\%eax \\
\hline Original Code & if(!driver->major) \{ \\
\hline Modified Code & if(!*(\&driver->major+0x1)) \{ \\
\hline
\end{tabular}

errors not manifested in our experiments. In this table, these errors are classified into 8 cases, based on the reason why they do not manifest themselves.

Corrected: "Corrected" indicates a situation in which an erroneous state is corrected by the Linux kernel. A typical example of this error is as follows. As shown in Table 5, a fault is injected to remove the initialization of oldpolicy. In the original code, oldpolicy is initialized to -1 . This error is corrected as follows.

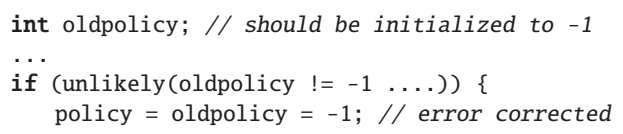

Not affecting: "Not affecting" indicates a situation where an erroneous state is not used by the kernel. For example, a local variable is corrupted but not used at all until the end of the function after the injection, as described in Table 5 (b). In this example, local variable all_pinned, which is not initialized, is not used in our experiments until the function returns.

Error processing omitted: "Error processing omitted" indicates a situation where the code for error processing is omitted. This error does not manifest itself during the experiments unless the omitted error processing becomes necessary. The detail of a typical example of this case is shown in Table 5 (c).

Incorrect warning: "Incorrect warning" indicates a situation where warning messages are displayed even though those messages should not be displayed. This is caused by the omission of conditional jumps that judge if warning messages should be (b) Not affecting

\begin{tabular}{|c|c|}
\hline Fault & INIT FAULT \\
\hline Memory Address & rebalance_tick+0xda \\
\hline Code Location & kernel/sched.c line: 2530 \\
\hline Original Instruction & movl $\$ \hat{\theta} x \boldsymbol{\theta}, \operatorname{\theta xfffffff\theta }(\% \mathrm{ebp})$ \\
\hline Modified nstruction & nop nop ... nop \\
\hline Original Code & int all_pinned $=0$; \\
\hline Modified Code & int all_pinned; \\
\hline
\end{tabular}

(d) Incorrect warning

\begin{tabular}{l|l}
\hline \hline Fault & BRANCH FAULT \\
\hline Memory Address & net_tx_action+0x37 \\
\hline Code Location & kernel/sched.c, line:2845 \\
\hline Original Instruction & je net_tx_action+0x55 \\
\hline Modified Instruction & nop nop \\
\hline Original Code & if (unlikely(! (x)) \{ \\
\hline Modified Code & deleted \\
\hline
\end{tabular}

(f) Aging

\begin{tabular}{l|l}
\hline \hline Fault & INVERSE FAULT \\
\hline Memory Address & mousedev_release+0x37 \\
\hline Code Location & drivers/input/mousedev.c, line:391 \\
\hline Original Instruction & jne mousedev_release+0x9a \\
\hline Modified Instruction & je mousedev_release+0x9a \\
\hline Original Code & if(!--list->mousedev->open $)\{$ \\
\hline Modified Code & if(--list->mousedev->open $)\{$
\end{tabular}

(h) Untraceable

\begin{tabular}{l|l}
\hline \hline Fault & INTERFACE FAULT \\
\hline Memory Address & rtnetlink_fill_ifinfo+0x2ec \\
\hline Code Location & net/core/rtnetlink.c, line:273 \\
\hline Original Instruction & mov 0x68(\%ebp), \%eax \\
\hline Modified Instruction & nop nop nop \\
\hline Original Code & u32 mtu $=$ dev->mtu; \\
\hline Modified Code & u32 mtu $=$ dev->broadcast; \\
\hline
\end{tabular}

displayed. The detail is shown in Table 5 (d).

Almost correct operation: "Almost correction operation" indicates a situation where the kernel behavior is slightly changed from the expected one but the kernel continues to run as normal. Most of these errors are related to scheduling parameters that affect the scheduling behavior of the kernel. In the example shown in Table 5 (e), the code for initializing local variable run_time is removed by fault injection. Since run_time is used to calculate the sleeping time of processes, it changes the scheduling behavior if set improperly. As shown below, even if run_time becomes erroneously large, the kernel code corrects the value. As a result, the kernel continues to run almost normally.

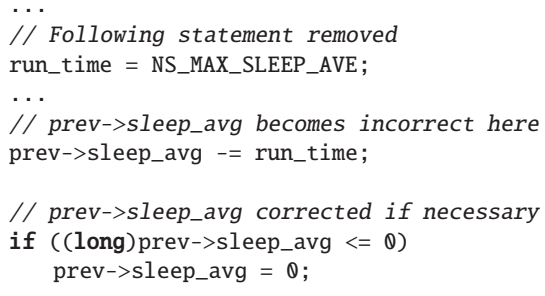

Aging: "Aging" indicates a situation where resource leakage occurs. Software aging is a serious problem but the aging errors seem not to manifest themselves during the short duration of fault injection experiments. An example of aging is shown in Table 5 (f). Before the fault injection, a reference counter is checked and the resource for a mouse device is released in this clause. Although this environment does not use mouse devices, 
the unreleased memory might pressure the kernel memory.

Lucky: "Lucky" indicates a situation where an error is activated but happens to cause nothing wrong. For example, INIT FAULT removes code for initializing a local variable to zero, whose value happens to be zero. Another example (shown in Table $5(\mathrm{~g})$ ) is from tty_register_driver, which is used to register a new major device. A PTR FAULT is injected into this function. In this case, the major device number of the new device becomes an unexpected number but the operation itself continues normally.

Untraceable: There are 11 cases in which we cannot trace error propagation completely. The faults are injected into the code for the socket management, and corrupt packet headers to be sent out to network. This example is shown in Table $5(\mathrm{~h})$. The actual operations of sending out the packets are performed asynchronously. So, we cannot trace the sending-out operations with the kernel debugger. We carefully observe the network behavior of the target machine but notice nothing in particular. This is probably because the packets with incorrect headers are destroyed somewhere deeper in network drivers. As a result, this type of errors does not manifest themselves.

\section{Discussion}

Our findings through the experiments are threefold. First, the Linux kernel is coded in a defensive way. This means that the Linux kernel frequently checks the integrity of function arguments, return values, and other important variables. This defensive coding style would have been introduced to ease debugging and diagnosis of failures. A typical example of the defensive coding in Linux is the use of BUG_ON macro, which checks the integrity of the kernel internal states. The use of BUG_ON aids in early error detections to prevent error propagation over the entire kernel. One interesting direction towards more resilient Linux is to develop a systematic method that determines the locations where BUG_ON macros are inserted and conditions given to those macros. Current static analysis tools are expected to give invaluable hints on the locations and conditions of BUG_ON macros. We show the Linux kernel that is used in this work is coded in a defensive way in terms of checking errors frequently. However, comparing the various analyses with different Linux kernel versions or systems software will show us other defensive ways of coding which is not discussed in this work.

Second, the scope of error propagation is mostly process-local in Linux. As you can see from our experimental results, most of the activated faults are process-local and do not propagate outside the contexts of faulty processes. This implies that the Linux can be rejuvenated without reboots with high probability. If an error does not propagate outside the context of the faulty process, the kernel states (including global data structures and other processes' contexts) are consistent. Thus, we can recover a consistent kernel state simply by revoking the context of the faulty process.

From our experiments, we have learned that our definition of "process-local" and "kernel-global" is somewhat ambiguous and there is room for further discussion. For example, some errors that cause software aging can be viewed as kernel-global because a resource leakage of a process affects all the other processes in the system. On the other hand, those errors can be viewed as process-local because no global data structures are corrupted; all processes are viewing consistent image of global data structures.

Finally, the global propagation of errors occurs in lower rates ( $16 \%$ of the failures). This is probably due to the nature of the defensive programming style in the Linux kernel. Data integrity is checked again and again before the data is written to global data structures.

One interesting finding in our investigation is that even if a global data structure is corrupted, the corrupted data cannot be accessed from the processes other than the faulty process. This is because global data structures are usually protected with an exclusive lock to avoid concurrent access to them. When a faulty process corrupts a global data structure, it often causes a segmentation fault before it exits the critical section. As a result, no other processes can access to the corrupted data structures.

This finding suggests that a new style of defensive programming. If a faulty process is revoked with a lock acquired, other processes cannot proceed because they cannot acquire the lock. To avoid this, when a faulty process with some locks acquired is revoked, the kernel should release the locks. Note that this design of the kernel increases the possibility that an error is propagated outside the faulty process context through corrupted global data structures. To prevent other processes from accessing corrupted data structures, when a process enters a critical section previously locked by a faulty process, it should check the integrity of the global data structures. If the integrity is confirmed, the process can proceed to access the data structures. Otherwise, the process tries to cure the corrupted data structures. If it succeeds, the process can proceed normally. If it fails, the process gives up accessing the data and calls panic to crash the kernel.

\section{Conclusion}

This paper investigates the Linux behavior under software faults. Our objective of this study is to gain some insight into 1) defensive coding style, 2) reboot-less rejuvenation, and 3) general recovery mechanisms of the Linux kernel. In particular, this paper focuses on the analysis on the scope of error propagation. If an error propagates inside the context of the faulty process, it is called process-local. If an error propagates outside the context of the faulty process, it is called kernel-global. To this end, we conduct an experimental campaign of fault injection on Linux. Since our focus is on software faults (in other words, software bugs), we use an existing software fault injector especially designed for injecting kernel-level software faults. It injects low- and high-level software faults. It is widely used in the OS research community. The major findings include:

- The Linux kernel is coded in a defensive way. It frequently checks the integrity of function arguments, return values, and other important variables. This style of coding contributes the resilience to activated faults. In particular, it contributes to lower rates of error manifestation (36\% of the fault activation) and global propagation (16\% of the failures). In our experiments, activated errors are often corrected or mitigated to avoid serious failures. This is because 
the Linux kernel checks data integrity again and again before updating global data structures.

- The scope of error propagation is mostly process-local in Linux. This implies that Linux can be rejuvenated without reboots with high probability. If an error does not propagate outside the context of a faulty process, time-consuming reboots can be avoided by revoking a faulty context because the kernel does not need to verify every kernel state. This result suggests that the kernel can recover from some failures by revoking a faulty context and undoing its resource acquisitions, for instance.

- Global propagation of errors occurs with low probability. Interestingly, even if a global data structure is corrupted, the corrupted data cannot be accessed from the other processes if the faulty process is killed within a critical section.

We believe our results of experimental fault injection suggest many directions of further research. As discussed in Section 6, we can take various approaches to improve the dependability of the Linux kernel. First, we expect that a tool for effectively inserting BUG_ON macros are required. Second, the Linux kernel can be rejuvenated without reboots with high probability. A mechanism that distinguishes a situation that can be recovered without reboots needs to be developed. Finally, the kernel can be recovered from kernel-global errors if we develop a sophisticated mechanism of handling errors in critical sections.

\section{References}

[1] Palix, N., Thomas, G., Saha, S., Calvés, C., Lawall, J. and Muller, G.: Faults in Linux: Ten Years Later, Proc. ACM International Conference on Architectural Support for Programming Languages and Operating Systems (ASPLOS'11), pp.305-318 (2011).

[2] Yoshimura, T., Yamada, H. and Kono, K.: Is Linux Kernel Oops Useful Or Not?, Proc. 8th Workshop on Hot Topics in System Dependability (HotDep'12) (2012).

[3] Ng, W.T. and Chen, P.M.: The Systematic Improvement of Fault Tolerance in the Rio File Cache, Proc. 29th Symposium on Fault-Tolerant Computing (FTCS '99), pp.76-83 (1999).

[4] Swift, M.M., Bershad, B.N. and Levy, H.M.: Improving the Reliability of Commodity Operating Systems, Proc. 19th ACM Symposium on Operating Systems Principles (SOSP '03), pp.207-222 (2003).

[5] Swift, M.M., Annamalai, M., Bershad, B.N. and Levy, H.M.: Recoverying Device Drivers, Proc. 6th USENIX Symposium on Operating Systems Design and Implementation (OSDI'04), pp.1-16 (2004).

[6] Depoutovitch, A. and Stumm, M.: Otherworld - Giving Applications a Change to Servive OS Kernel Crashes, Proc. 5th European Conference on Computer Systems (EuroSys '10), pp.181-194 (2010).

[7] Yamakita, K., Yamada, H. and Kono, K.: Phase-based Reboot: Reusing Operating System Execution Phases for Cheap Reboot-based Recovery, Proc. 41st Annual IEEE/IFIP International Conference on Dependable Systems and Networks (DSN'11), pp.169-180 (2011).

[8] Ng, W.T. and Chen, P.M.: The Design and Verification of the Rio File Cache, IEEE Trans. Comput., Vol.50, No.4, pp.322-337 (2001).

[9] Gu, W., Kalbarczyk, Z., Iyer, R.K. and Yang, Z.: Characterization of Linux Kernel Behavior under Errors, Proc. 2003 IEEE International Conference on Dependable Systems and Networks (DSN '03), pp.459468 (2003).

[10] Gu, W., Kalbarczyk, Z. and Iyer, R.K.: Error Sensitivity of the Linux kernel Executing on PowerPC G4 and Pentium 4 Processors, Proc. 4th IEEE International Conference on Dependable Systems and Networks (DSN '04), pp.887-896 (2004).

[11] Chen, D., Jacques-Silva, G. and Mealey, B.: Error Behavior Comparison of Multiple Compuing System: A Case Study Ui Linux on Pentium, Solaris on SPARC, and AIX and POWER, Proc. 14th IEEE Pacific Rim International Symposium On Dependable Computing (PRDC '08), pp.339-346 (2008).

[12] Patterson, D.A.: An Introduction to Dependability, ;login; Vol.27, No.4 (2002).

[13] Yoshimura, T., Yamada, H. and Kono, K.: Can Linux be Rejuvenated without Reboots?, Proc. IEEE 3rd International Workshop on Software Aging and Rejuvenation (WoSAR '11) (2011).

[14] Pham, C., Chen, D., Kalbarczyk, Z. and Iyer, R.K.: CloudVal: A framework for validation of virtualization environment in cloud infrastructure, Proc. 41st Annual IEEE/IFIP International Conference on Dependable Systems and Networks (DSN'11), pp.189-196 (2011).

[15] Duraes, J. and Madeira, H.S.: Emulation of Software Faults: A Field Data Study and a Practical Approach, IEEE Trans. Softw. Eng., Vol.32, No.11, pp.849-867 (2006).

[16] Cotroneo, D., Lanzaro, A., Natella, R. and Barbosa, R. Experimental Analysis of Binary-Level Software Fault Injection in Complex Software, Proc. IEEE 9th European Dependable Computing Conference (EDCC '12) (2012).

[17] Chou, A., Yang, J., Chelf, B., Hallem, S. and Engler, D.: An Empirical Study of Operating Systems Errors, Proc. 18th ACM Symposium on Operating Systems Principles (SOSP '01), pp.73-88 (2001).

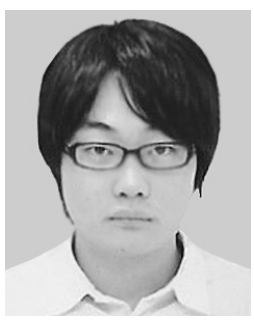

Takeshi Yoshimura was born in 1988. He received his B.E. degree from the Department of Information and Computer Science at Keio University. $\mathrm{He}$ is currently a graduate student at the School of Science for Open and Environmental Systems at Keio University. His research interests include dependable systems, operating systems and virtualization.

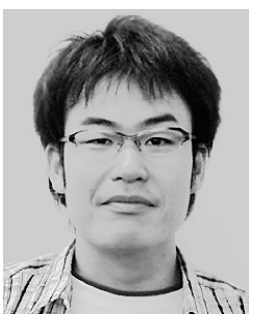

Hiroshi Yamada was born in 1981. He received his B.E. and M.E. degrees from the University of Electro-communications in 2004 and 2006, respectively. He received his Ph.D. degree from Keio University in 2009. He is currently an associate professor of the Division of Advanced Information Technology \& Computer Science at Tokyo University of Agriculture and Technology. His research interests include operating systems, virtualization, and dependable systems. He is a member of ACM, USENIX and IEEE/CS.

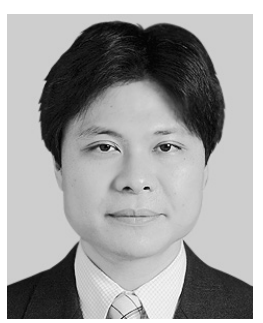

Kenji Kono received his B.Sc. degree in 1993, M.Sc. degree in 1995, and Ph.D. degree in 2000, all in computer science from the University of Tokyo. He is an associate professor of the Department of Information and Computer Science at Keio University. His research interests include operating systems, system software, and Internet security. $\mathrm{He}$ is a member of IEEE/CS, ACM and USENIX. 